This document is published in:

Physics Letters A (1997), 226 (1-2), pp. 7-13.

DOI: 10.1016/S0375-9601(96)00911-5

(C) 1997 Elsevier B.V. 


\title{
Asymptotic formula for the quantum entropy of position in energy eigenstates
}

\author{
Jorge Sánchez-Ruiz ${ }^{1}$ \\ Departament de Física Fonamental, Universitat de Barcelona. Diagonal 647, 08028 Barcelona. Spain
}

Received 5 December 1995; revised manuscript received 18 November 1996; accepted for publication 28 November 1996

Communicated by P.R. Holland

\begin{abstract}
The asymptotic formula $S_{\mathrm{Q}} \sim S_{\mathrm{C}}-1+\ln 2$ is obtained for the information entropy in position space $S_{\mathrm{Q}}$ of one-dimensional quantum systems in energy eigenstates, where $S_{\mathrm{C}}$ is the position entropy corresponding to a microcanonical ensemble of analogous classical systems having the same energy. This result is analytically and numerically verified for several simple systems.
\end{abstract}

PACS: $03.65 . \mathrm{Sq} ; 02.30 . \mathrm{Mv}$

Keywords: Entropy: Correspondence principle; WKB approximation

\section{Introduction}

Bohr's correspondence principle states, roughly speaking, that quantum mechanics reduces to classical mechanics in the large quantum-number limit. In the framework of modern quantum mechanics, this principle is often illustrated by noting that the local average of the quantum probability density of position $P_{Q}(x)=\left|\psi_{n}(x)\right|^{2}$ in the energy eigenstate $\psi_{n}$ for a system with an infinite discrete spectrum $\left\{E_{n}\right\}$ approaches, in the limit of large $n$, the probability density $P_{\mathrm{C}}(x)$ corresponding to a microcanonical ensemble of analogous classical systems with energy $E=E_{n}[1-5]$, which is sometimes referred to as the configuration correspondence principle [2]. It follows that the expected value of any observable $F(x)$ in the eigenstate $\psi_{n}$ approaches the corresponding

\footnotetext{
'E-mail: jsanchez@hermes.ffn.ub.es.
}

average for the analogous classical system having the same energy,

$\langle F(x)\rangle_{\mathrm{Q}} \sim\langle F(x)\rangle_{\mathrm{C}}$.

However, as explained below, Eq. (1) does not hold when the function $F(x)$ depends explicitly on the probability density $P(x)$. A physically interesting example is the Boltzmann-Shannon information entropy of position,

$S=-\int P(x) \ln P(x) \mathrm{d} x=\langle-\ln P(x)\rangle$,

which measures the uncertainty in the localization of the particle in position space. The quantum entropy $S_{\mathrm{Q}}=\left\langle-\ln P_{\mathrm{Q}}(x)\right\rangle$ and its counterpart in momentum space have been used during recent years to discuss a wide range of quantum-mechanical problems, such as the mathematical formulation of the positionmomentum uncertainty relation $[6,7]$ and spreading 
of wave packets [8], the study of single-particle densities of fermionic systems [9], approximate calculations of energy eigenvalues and eigenstates by means of the maximum-entropy principle [10], and time evolution of chemical reactions [11].

The analytical value of $S_{\mathrm{Q}}$ is only known in a few particular cases, even for simple systems as the harmonic oscillator and hydrogen atom [12], which has attracted interest to its approximate calculation, specially for very excited or Rydberg states (i.e., for large $n$ ). Such asymptotic formulas for $S_{\mathrm{Q}}$ have been obtained in the case when $P_{\mathrm{Q}}(x) \propto p_{n}^{2}(x) w(x)$, where $p_{n}(x)$ are orthogonal polynomials with respect to the weight $w(x)$ on a finite interval [13], or Freud orthogonal polynomials $\left(w(x)=\exp \left(-|x|^{m}\right), m>0\right)$ on the whole real axis $[14,15]$, but no general result of this kind has been found up to now for arbitrary quantum systems. In this Letter we show that, in the simplest case of a one-dimensional system whose Hamiltonian is of the form

$H=\frac{p^{2}}{2 m}+V(x)$,

where, at least for large $n$, there are two (and no more than two) classical turning points for $E=E_{n}$ (see Section 2 for the precise definition of turning points), a very simple reasoning based on the WKB approximation leads to the general asymptotic formula

$S_{\mathrm{Q}} \sim S_{\mathrm{C}}-1+\ln 2$,

which implies that the difference between $S_{\mathrm{Q}}$ and the corresponding classical entropy $S_{\mathrm{C}}=\left\langle-\ln P_{\mathrm{C}}(x)\right\rangle$ does tend to a nonzero constant, at variance with the usual statement of the correspondence principle (1). Eq. (4) provides a useful approximation to $S_{\mathrm{Q}}$ for large $n$, which can be applied for any Hamiltonian of the form (3) without requiring the previous knowledge of the corresponding quantum eigenfunctions.

The differential entropy $S$ defined by Eq. (2) contains the logarithm of the probability density $P(x)$, which has a dimension of inverse length, so that it is measured in (say) "log-meters". Although this may seem somewhat unusual, it only means that the entropy changes by an additive constant when a change of scale is made, i.e. $S$ changes to $S-\ln a$ if we replace $P(x)$ by $a P(a x), a>0[16]$. This transformation rule takes a more natural form (the same that obeys, e.g., the standard deviation [16]) for the exponential of the entropy, a nonnegative quantity which has a dimension of length and is a good measure of the width of the curve $P(x)$. For example, $\exp (S)=L$ when $P(x)$ is a uniform distribution extended over an interval of length $L$, while a simple variational calculation shows that $\exp (S)<L$ for any nonuniform distribution vanishing outside that interval, and it tends to zero when $P(x)$ tends to a Dirac delta distribution. Thus it appears more convenient to consider $\exp (S)$ rather than $S$ itself as a measure of uncertainty for continuous distributions. However, such a rescaling procedure is purely conventional, so that both $\exp (S)$ and $S$ represent in fact the same notion of uncertainty [16]. It is also worth noting that differences between any two entropies, such as $S_{\mathrm{Q}}-S_{\mathrm{C}}$, are dimensionless quantities.

On the other hand, actual physical measurements of any continuous observable such as position are always performed by means of measuring devices that have finite resolution. This means that the continuous spectrum of the position observable is partitioned into a countable set of intervals (or "bins", in the terminology of Ref. [17]) of length $\Delta x>0$ (for the sake of simplicity, we assume the resolution of the measuring device to be uniform). The probability $P_{i}^{(\Delta x)}$ of finding the outcome of the position measurement to have a value in the $i$ th interval, $(\Delta x)_{i}$, is the integral of $P(x) \mathrm{d} x$ over this interval, and the entropy $S^{(\Delta x)}$ associated to the discrete probability distribution $\left\{P_{i}^{(\Delta x)}\right\}$ is $[17,18]$

$$
\begin{aligned}
& S^{(\Delta x)}=-\sum_{i}\left(\int_{(\Delta x)_{i}} P(x) \mathrm{d} x\right) \\
& \times \ln \left(\int_{(\Delta x)_{i}} P(x) \mathrm{d} x\right) .
\end{aligned}
$$

Unlike the differential entropy $S$, the finite resolution entropy $S^{(\Delta x)}$ is a dimensionless quantity. It is nonnegative, $S^{(\Delta x)} \geqslant 0$, and also satisfies the inequality [18]

$S^{(\Delta x)} \geqslant S-\ln (\Delta x)$,

which becomes an equality in the limit $\Delta x \rightarrow 0$. Therefore, in this limit, the difference between the finite resolution entropies of any two probability densities coincides with the difference between the corresponding differential entropies, i.e., $S_{2}^{(\Delta x)}-S_{1}^{(\Delta x)}=$ 
$S_{2}-S_{1}$. In particular, the asymptotic formula (4) is also valid for the quantum and classical entropies $S^{(\Delta x)}$

$S_{\mathrm{Q}}^{(\Delta x)} \sim S_{\mathrm{C}}^{(\Delta x)}-1+\ln 2, \quad \Delta x \rightarrow 0$.

The derivation of Eq. (4) is carried out in Section 2, and this result is verified in Section 3 for the particular cases of the particle in a box (infinite potential well), the linear potential, and the harmonic oscillator. Finally, in Section 4 some concluding remarks are given and several open problems are pointed out.

\section{Derivation of the asymptotic formula (4)}

The motion of a classical particle with Hamiltonian (3) and (constant) energy $E$ is periodic between the turning points $x_{-}$and $x_{+}\left(x_{-}<x_{+}\right)$, where the speed $v=p / m$ of the particle reduces to zero,

$V\left(x_{-}\right)=V\left(x_{+}\right)=E$,

and there exists a nonvanishing force $-V^{\prime}(x)$ that causes the particle to move towards the right at $x=$ $x_{-}$, and towards the left at $x=x_{+}$,

$V^{\prime}\left(x_{-}\right)<0, \quad V^{\prime}\left(x_{+}\right)>0$.

The probability $P_{\mathrm{C}}(x) \mathrm{d} x$ of finding the particle in the region between $x$ and $x+\mathrm{d} x$ is proportional to the amount of time $\mathrm{d} t$ the particle spends in that region during one traversal of the potential well (from, say, $x_{-}$to $x_{+}$), which in turn is inversely proportional to the speed $v=\mathrm{d} x / \mathrm{d} t$ at the point $x$ (or, equivalently, to the momentum $p=m u$ ) $[1-5,19]$. Using Eq. (3), it can be shown [5] that the normalized classical probability density for the position of the particle is given by

$P_{\mathrm{C}}(x)=\frac{1}{T} \sqrt{\frac{2 m}{E-V(x)}}$

within the classically allowed region $x_{-} \leqslant x \leqslant x_{+}$, where $T$ is the period of the motion. In terms of the reduced de Broglie wavelength,

$\lambda(x)=\frac{\hbar}{\sqrt{2 m[E-V(x)]}}$,
Eq. (10) reads

$P_{\mathrm{C}}(x)=\frac{2 m}{T \hbar} \lambda(x)$,

which shows that the functions $P_{C}(x)$ and $\lambda(x)$ are proportional to each other.

On the other hand, in the limit of large quantum numbers the quantum probability density $P_{\mathrm{Q}}(x)$ also vanishes outside the classical region $\left(x_{-}, x_{+}\right)$, while inside this region the WKB approximation gives $[1,4]$

$P_{\mathrm{Q}}(x) \sim N P_{\mathrm{C}}(x) \cos ^{2} \phi(x)$,

$\phi(x)=\int_{x_{-}}^{x} \frac{\mathrm{d} x^{\prime}}{\lambda\left(x^{\prime}\right)}-\frac{\pi}{4}$,

where $N$ is a normalization constant. For large $n$, the energy levels $E_{n}$ are determined by the WKB quantization rule $[1,4]$,

$\int_{x_{-}}^{x_{+}} \frac{\mathrm{d} x^{\prime}}{\lambda\left(x^{\prime}\right)}=\left(n+\frac{1}{2}\right) \pi$,

which enables us to write

$\phi(x)=\left(n+\frac{1}{2}\right) u(x)-\frac{1}{4} \pi$,

where $u(x)$ is a monotonically increasing function with $u\left(x_{-}\right)=0$ and $u\left(x_{+}\right)=\pi$. It follows from Eq. (15) that the function $\cos ^{2} \phi(x)$ has $n$ zeros, which are located at the points $x_{k}$ satisfying the condition

$u\left(x_{k}\right)=\frac{k+3 / 4}{n+1 / 2} \pi, \quad k=0,1, \ldots, n-1$.

Therefore, for large $n, \cos ^{2} \phi(x)$ is a very rapidly oscillating function and can be replaced by the average value

$\left\langle\cos ^{2} x\right\rangle=\frac{1}{\pi} \int_{0}^{\pi} \cos ^{2} x \mathrm{~d} x=\frac{1}{2}$

in the normalization integral for $P_{\mathrm{Q}}(x)$. A rigorous proof of this statement may be achieved by making the change of variable $u(x)=\theta$ and applying, with $g(\theta)=\cos ^{2} \theta$, the following theorem (Lemma 2.1 in Ref. [14]), 


$$
\begin{aligned}
& \int_{0}^{\pi} g(n \theta+\gamma(\theta)) f(\theta) \mathrm{d} \theta \\
& \sim \frac{1}{\pi} \int_{0}^{\pi} g(\theta) \mathrm{d} \theta \int_{0}^{\pi} f(\theta) \mathrm{d} \theta,
\end{aligned}
$$

where $g$ is a continuous function such that $g(\theta+\pi)=$ $g(\theta), f \in L^{1}([0, \pi])$, and $\gamma$ is a measurable and almost everywhere finite on $[0, \pi]$ function. We thus find $N \sim 2$, and, accordingly,

$$
P_{\mathrm{Q}}(x) \sim 2 P_{\mathrm{C}}(x) \cos ^{2} \phi(x) \text {. }
$$

The same reasoning enables us to prove Eq. (1) for any position-dependent observable $F(x)$,

$$
\begin{aligned}
& \langle F(x)\rangle_{\mathrm{Q}}=\int_{-\infty}^{\infty} P_{\mathrm{Q}}(x) F(x) \mathrm{d} x \\
& \sim 2 \int_{x_{-}}^{x_{+}} P_{\mathrm{C}}(x) F(x) \cos ^{2} \phi(x) \mathrm{d} x \\
& \sim \int_{x_{-}}^{x_{+}} P_{\mathrm{C}}(x) F(x) \mathrm{d} x=\langle F(x)\rangle_{\mathrm{C}} .
\end{aligned}
$$

However, this derivation assumes $F(x)$ to be a smoothly varying function, whose value can be considered as a constant during a period of $\cos ^{2} \phi(x)$. Therefore, it cannot be applied when $F(x)$ depends explicitly on the quantum probability density $P_{\mathrm{Q}}(x)$, thus being itself a rapidly oscillating function. In the particular case of the Boltzmann-Shannon information entropy, substitution for $P_{\mathrm{Q}}(x)$ from (19) into (2) gives

$$
\begin{aligned}
S_{\mathrm{Q}} & =-\int_{-\infty}^{\infty} P_{\mathrm{Q}}(x) \ln P_{\mathrm{Q}}(x) \mathrm{d} x \\
& \sim-2 \ln 2 \int_{x_{-}}^{x_{+}} P_{\mathrm{C}}(x) \cos ^{2} \phi(x) \mathrm{d} x \\
& -2 \int_{x_{-}}^{x_{+}} P_{\mathrm{C}}(x) \cos ^{2} \phi(x) \ln P_{\mathrm{C}}(x) \mathrm{d} x
\end{aligned}
$$

$$
-2 \int_{x_{-}}^{x_{+}} P_{\mathrm{C}}(x) \cos ^{2} \phi(x) \ln \cos ^{2} \phi(x) \mathrm{d} x .
$$

For large $n, \cos ^{2} \phi(x)$ can be replaced again by $\frac{1}{2}$ in the first and second integrals, while application of (18) with $g(\theta)=\cos ^{2} \theta \ln \cos ^{2} \theta, \theta=u(x)$, shows that $\cos ^{2} \phi(x) \ln \cos ^{2} \phi(x)$ can likewise be replaced in the last integral by the corresponding average value,

$$
\begin{aligned}
& \left\langle\cos ^{2} x \ln \cos ^{2} x\right\rangle=\frac{1}{\pi} \int_{0}^{\pi} \cos ^{2} x \ln \cos ^{2} x \mathrm{~d} x \\
& =\frac{1}{2}-\ln 2,
\end{aligned}
$$

which leads to Eq. (4). The same method could be used to find similar asymptotic relations for the expected values of other functions $F(x)$ explicitly depending on $P(x)$.

\section{Examples}

It is interesting to note that, for a particle in a box (infinite potential well) of length $L$, we have $[1,4]$

$$
\begin{aligned}
& P_{\mathrm{C}}(x)=\frac{1}{L}, \quad P_{\mathrm{Q}}(x)=\frac{2}{L} \sin ^{2}\left(\frac{n \pi x}{L}\right), \\
& 0 \leqslant x \leqslant L,
\end{aligned}
$$

so that the corresponding entropies are

$S_{\mathrm{C}}=\ln L, \quad S_{\mathrm{Q}}=\ln (2 L)-1$,

and the asymptotic relation (4) is thus shown to become an exact equality in this case.

As our second example we consider the linear potential $V(x)=V_{a}|x| / a$, with $V_{a}, a>0$, for which the quantum probability density is expressed in terms of the Airy function $[4,5]$,

$$
\begin{aligned}
& P_{\mathrm{Q}}(x)=\left|\psi_{n}(x)\right|^{2}=N_{n} \mathrm{Ai}^{2}\left(\alpha\left(x-\beta_{n}\right)\right), \\
& \alpha=\left(\frac{2 m V_{a}}{\hbar^{2} a}\right)^{1 / 3}, \quad \beta_{n}=\frac{a E_{n}}{V_{a}}, \quad x \geqslant 0 .
\end{aligned}
$$

It suffices to consider only the odd-parity eigenfunctions, whose corresponding eigenvalues are determined by the condition 
$\operatorname{Ai}\left(-\epsilon_{n}\right)=0, \quad \epsilon_{n}=\left(\frac{2 m a^{2}}{\hbar^{2} V_{a}^{2}}\right)^{1 / 3} E_{n}=\alpha \beta_{n}$.

The analytical value of the normalization constant $N_{n}$ is then [20]

$N_{n}=\frac{\alpha}{2}\left(\int_{-\epsilon_{n}}^{\infty} \mathrm{Ai}^{2}(t) \mathrm{d} t\right)^{-1}=\frac{\alpha}{2 \mathrm{Ai}^{\prime 2}\left(-\epsilon_{n}\right)}$,

so that we have

$$
\begin{aligned}
S_{\mathrm{Q}} & =-\int_{-\infty}^{\infty} P_{\mathrm{Q}}(x) \ln P_{\mathrm{Q}}(x) \mathrm{d} x \\
& =\ln \left(\frac{2 \mathrm{Ai}^{2}\left(-\epsilon_{n}\right)}{\alpha}\right) \\
& -\frac{1}{\mathrm{Ai}^{\prime 2}\left(-\epsilon_{n}\right)} \int_{-\epsilon_{\|}}^{\infty} \mathrm{Ai}^{2}(t) \ln \mathrm{Ai}^{2}(t) \mathrm{d} t .
\end{aligned}
$$

On the other hand, the normalized classical probability density for $E=E_{n}$ is

$P_{\mathrm{C}}(x)=\frac{1}{4 \beta_{n}}\left(1-\frac{|x|}{\beta_{n}}\right)^{-1 / 2}$,

and the corresponding classical entropy is given by

$S_{\mathrm{C}}=-\int_{-\beta_{n}}^{\beta_{n}} P_{\mathrm{C}}(x) \ln P_{\mathrm{C}}(x) \mathrm{d} x=\ln \left(\frac{4 \epsilon_{n}}{\alpha}\right)-1$.

The first ten zeros of the Airy function may be found in Ref. [21], while for $n>10$ we can calculate $\epsilon_{n}$ by means of the asymptotic expansion $[20,21]$

$\epsilon_{n} \sim \mathrm{f}\left(\frac{3 \pi(4 n-1)}{8}\right)$,

$f(z)=z^{2 / 3}\left(1+\frac{5}{48} z^{-2}-\frac{5}{36} z^{-4}+\cdots\right)$,

whose leading term is the WKB approximate value given by (14) [4]. The resulting numerical values of the classical and quantum entropies, as well as their difference, are displayed for several values of $n$ in Table 1. From the last column of this table, we conjecture that (4) can be improved in this case to
Table 1

Numerical values of the classical entropy $S_{C}$ for $\alpha=1$, the quantum entropy $S_{\mathrm{Q}}$ for $\alpha=1$, the difference $S_{\mathrm{C}}-S_{\mathrm{Q}}$, and $k_{n}=$ $n^{1 / 3}\left(1-\ln 2-S_{\mathrm{C}}+S_{\mathrm{Q}}\right)$ for the potential $V(x)=V_{a}|x| / a$ in several odd-parity energy eigenstates $\psi_{n}$

\begin{tabular}{rllll}
\hline$n$ & $S_{C}(\alpha=1)$ & $S_{\mathrm{Q}}(\alpha=1)$ & \multicolumn{1}{l}{$S_{\mathrm{C}}-S_{\mathrm{Q}}$} & \multicolumn{1}{l}{$k_{n}$} \\
\hline 1 & 1.235636 & 1.710184 & -0.474548 & 0.781400 \\
2 & 1.794338 & 2.076628 & -0.282290 & 0.742273 \\
3 & 2.094774 & 2.294433 & -0.199659 & 0.730517 \\
4 & 2.301260 & 2.451056 & -0.149796 & 0.724885 \\
5 & 2.458728 & 2.573862 & -0.115134 & 0.721587 \\
10 & 2.937985 & 2.963090 & -0.025105 & 0.715182 \\
20 & 3.408539 & 3.364019 & 0.044520 & 0.712082 \\
30 & 3.681649 & 3.603638 & 0.078011 & 0.711066 \\
40 & 3.874834 & 3.775750 & 0.099083 & 0.710561 \\
50 & 4.024433 & 3.910375 & 0.114059 & 0.710260 \\
100 & 4.488203 & 4.334242 & 0.153961 & 0.709660 \\
200 & 4.951136 & 4.765582 & 0.185554 & 0.709362 \\
300 & 5.221724 & 5.020821 & 0.200903 & 0.709263 \\
400 & 5.413651 & 5.203053 & 0.210598 & 0.709213 \\
500 & 5.562496 & 5.344995 & 0.217501 & 0.709183 \\
1000 & 6.024761 & 5.788821 & 0.235940 & 0.709124 \\
2000 & 6.486943 & 6.236371 & 0.250572 & 0.709095 \\
3000 & 6.757281 & 6.499593 & 0.257688 & 0.709085 \\
4000 & 6.949082 & 6.686899 & 0.262184 & 0.709080 \\
5000 & 7.097853 & 6.832467 & 0.265386 & 0.709077 \\
10000 & 7.559968 & 7.286027 & 0.273941 & 0.709071 \\
\hline
\end{tabular}

$S_{\mathrm{Q}} \sim S_{\mathrm{C}}-1+\ln 2+\frac{k}{n^{1 / 3}}+o\left(\frac{1}{n^{1 / 3}}\right)$,

$k \approx 0.709$.

Finally, for the harmonic oscillator potential $V(x)=$ $\frac{1}{2} m \omega^{2} x^{2}$, the quantum probability density is $[1,4,5]$

$$
\begin{gathered}
P_{\mathrm{Q}}(x)=\left|\psi_{n}(x)\right|^{2}=\alpha\left(2^{n} n ! \sqrt{\pi}\right)^{-1} H_{n}^{2}(\alpha x) \mathrm{e}^{-\alpha^{2} x^{2}}, \\
\alpha=\left(\frac{m \omega}{\hbar}\right)^{1 / 2},
\end{gathered}
$$

where $H_{n}(x)$ are Hermite polynomials, while, for $E=$ $E_{n}=\left(n+\frac{1}{2}\right) \hbar \omega$, one has the classical probability density

$P_{\mathrm{C}}(x)=\frac{1}{\pi \sqrt{A_{n}^{2}-x^{2}}}, \quad A_{n}=\frac{\sqrt{2 n+1}}{\alpha}$,

so that the classical entropy is

$S_{\mathrm{C}}=-\int_{-A_{n}}^{A_{n}} P_{\mathrm{C}}(x) \ln P_{\mathrm{C}}(x) \mathrm{d} x=\ln \left(\frac{\pi \sqrt{2 n+1}}{2 \alpha}\right)$. 
The asymptotic formula for $S_{\mathrm{Q}}$ given by (4) and (33),

$S_{\mathrm{Q}} \sim \ln \left(\frac{\pi \sqrt{2 n+1}}{\alpha}\right)-1 \sim \ln \left(\frac{\pi \sqrt{2 n}}{\alpha}\right)-1$,

coincides with that obtained by means of the theory of strong asymptotics of Freud orthogonal polynomials, which include Hermite polynomials as a particular case $[14,15]$, and also agrees with the exact numerical values of $S_{\mathrm{Q}}$ for $n \gg 1[12,15]$. An independent derivation of Eq. (34) on the basis of the asymptotic relation between $P_{\mathrm{Q}}(x)$ and $P_{\mathrm{C}}(x)$ for the harmonic oscillator has been given very recently by Majerník and Opatrný [22].

\section{Summary and open problems}

In summary, we have derived the general asymptotic formula (4) for the quantum entropy of position of one-dimensional systems in energy eigenstates in terms of the corresponding classical entropy (i.e., the entropy corresponding to a microcanonical ensemble of analogous classical systems having the same energy). This result was obtained by means of the WKB approximation for systems whose Hamiltonian is of the form (3) and only have two classical turning points for $E=E_{n}$, at least for large $n$.

Eq. (4) has been checked for three simple systems, namely the particle in a box (where the asymptotic formula becomes an exact identity), the linear potential, and the harmonic oscillator. In particular, the asymptotic formula for the harmonic oscillator (34) coincides with that obtained by means of the theory of strong asymptotics of orthogonal polynomials $[14,15]$. This result strongly suggests the mathematical equivalence of these two seemingly very different approaches, although the problem of finding a rigorous proof of this equivalence remains open. On the other hand, it is worth noting that, when derived from (4), asymptotic formulas such as (34) are shown to be a simple consequence of the correspondence principle (1), with the correction term $1-\ln 2$ arising due to the explicit dependence of $F(x)=-\ln P(x)$ on the probability density, which provides them with a more clear physical interpretation.

The entropy $S^{(\Delta x)}$ defined by Eq. (5) illustrates the transition between Eqs. (1) and (4). The average distance between two consecutive zeros of $\cos ^{2} \phi(x)$ is approximately equal to $\left(x_{+}-x_{-}\right) / n$ for large $n$ ( see Section 2). If this distance is much less than $\Delta x$, the function $\cos ^{2} \phi(x)$ oscillates very rapidly over each interval $(\Delta x)_{i}$, so that it can be replaced by the average value $\frac{1}{2}$ in the calculation of $S^{(\Delta x)}$ for the quantum probability density given by the WKB approximation (Eq. (19)). We thus have

$S_{\mathrm{Q}}^{(\Delta x)} \sim S_{\mathrm{C}}^{(\Delta x)}, \quad \Delta x \gg \frac{x_{+}-x_{-}}{n}$,

while in the opposite case we can make use of Eq. (7), which may be written more precisely as

$S_{\mathrm{Q}}^{(\Delta x)} \sim S_{\mathrm{C}}^{(\Delta x)}-1+\ln 2, \quad \Delta x \ll \frac{x_{+}-x_{-}}{n}$.

For a $D$-dimensional system whose Hamiltonian is completely separable in Cartesian coordinates,

$H=\sum_{i=1}^{D} H_{i}\left(x_{i}, p_{i}\right)$,

the quantum and classical probability densities for position are products of $D$ one-dimensional densities. Since the entropy $S$ is now defined by the $D$ dimensional generalization of Eq. (2), in this case both $S_{\mathrm{Q}}$ and $S_{\mathrm{C}}$ are sums of $D$ one-dimensional entropies. Therefore, if every one-dimensional Hamiltonian $H_{i}$ has the form (3), so that Eq. (4) is valid for each coordinate, in the limit $n_{i} \gg 1, i=1, \ldots, D$, we have the asymptotic formula

$S_{\mathrm{Q}} \sim S_{\mathrm{C}}-D(1-\ln 2)$,

which becomes an exact equality for the $D$ dimensional infinite potential well. However, the more involved problem of finding the asymptotic relation between quantum and classical entropies of position for systems whose Hamiltonian is not of the form (37) (or (3) in the one-dimensional case) has not yet been solved.

Another open problem is that of whether a counterpart of Eq. (4) does exist in momentum space. Owing to the symmetry between position and momentum in the harmonic oscillator Hamiltonian, both classical and quantum momentum entropies coincide, up to an additive constant, with the respective position entropies, which implies that then Eq. (4) is also valid for momentum entropies. Leaving aside this special 
case, however, no simple asymptotic relation such as (19) between quantum and classical momentum probability densities is known, so that the problem of obtaining a general asymptotic formula for the quantum entropy of momentum in energy eigenstates remains open.

\section{Acknowledgement}

The author thanks R.J. Yáñez and J.S. Dehesa for interesting discussions and for supplying me preprints of Refs. [14,15], and the anonymous referees for useful suggestions. This work was supported by a grant from the Fundació Aula (Barcelona, Spain).

\section{References}

[1] L.1. Schiff, Quantum mechanics, 3rd Ed. (McGraw-Hill, Tokyo, 1968);

R.B. Leighton, Principles of modern physics (McGraw-Hill, New York, 1959);

C. Cohen-Tannoudji, B. Diu and F. Laloë, Quantum mechanics (Wiley, New York, 1977).

|2| R.L. Liboff, Phys. Today 37 (1984) 50.

|3| G.G. Cabrera and M. Kiwi, Phys. Rev. A 36 (1987) 2995.

|4| A. Galindo and P. Pascual, Quantum mechanics (Springer, Berlin, 1991).
[5] R.W. Robinett, Am. J. Phys. 63 (1995) 823.

[6] W. Beckner, Amn. Math. 102 (1975) 159

[7] I. Bialynicki-Birula and J. Mycielski, Commun. Math. Phys. 44 (1975) 129.

|8| M. Grabowski, J. Math. Phys. 22 (1981) 303; Rep. Math. Phys. 24 (1986) 327.

19| S.R. Gadre and R.D. Bendale, Phys. Rev. A 36 ( 1987) 1932; J.C. Angulo and J.S. Dehesa, J. Chem. Phys. 97 (1992) 6485.

[10] N. Canosa, A. Plastino and R. Rossignoli, Phys. Rev. A 40 (1989) 519;

N. Canosa, R. Rossignoli and A. Plastino, Phys. Rev. A 43 (1991) 1145

[11] N. Balakrishnan and N. Sathyamurthy, Chem. Phys. Lett. 164 (1989) 267.

[12] R.J. Yáñez, W. Van Assche and J.S. Dehesa, Phys. Rev. A 50 (1994) 3065.

[13] A.I. Aptekarew, J.S. Dehesa and R.J. Yáñez, J. Math. Phys. 35 (1994) 4423.

[14] A.I. Aptekarev, V.S. Buyarov and J.S. Dehesa, Russian Acad. Sci. Sbornik Math. 82 (1995) 373 .

[15] W. Van Assche, R.J. Yáñez and J.S. Dehesa, J. Math. Phys. 36 (1995) 4106.

[16] J.B.M. Uffink, Measures of uncertainty and the uncertainty principle, Ph.D. thesis, University of Utrecht (1990)

[17] M.H. Partovi, Phys. Rev. Lett. 50 (1983) 1883.

[18] I. Bialynicki-Birula, Phys. Lett. A 103 (1984) 253.

[19] J. Peslak. Jr., Am. J. Phys. 47 (1979) 39

[20] F.W.J. Olver, Asymptotics and special functions (Academic Press, New York, 1974)

[21] M. Abramowitz and I.A. Stegun, eds., Handbook of mathematical functions (Dover, New York, 1965).

|22| V. Majerník and T. Opatrný. J. Phys. A 29 (1996) 2187. 